\title{
Immune-Checkpoint-Inhibitor-Induced Severe Autoimmune Encephalitis Treated by Steroid and Intravenous Immunoglobulin
}

\author{
Ahwon Kim ${ }^{\mathrm{a}}$ \\ Bhumsuk Keam ${ }^{\mathrm{b}}$ \\ Hyeon Cheun ${ }^{\mathrm{C}}$ \\ Soon-Tae Lee ${ }^{a}$ \\ Hyung Seok Gook ${ }^{\mathrm{a}}$ \\ Moon-Ku Han ${ }^{\mathrm{d}}$ \\ Departments of Neurology and \\ 'Internal Medicine, \\ Seoul National University Hospital, \\ Seoul, Korea \\ 'Seoul National University, \\ College of Medicine, Seoul, Korea \\ ${ }^{\mathrm{d} D e p a r t m e n t}$ of Neurology, \\ Seoul National University, \\ College of Medicine, Seoul, Korea
}

Received September 11, 2018

Revised November 12, 2018

Accepted November 13, 2018

\section{Correspondence}

Moon-Ku Han, MD, PhD

Department of Neurology,

Seoul National University,

College of Medicine,

82 Gumi-ro 173beon-gil, Bundang-gu,

Seongnam 13620, Korea

Tel +82-31-787-7464

Fax +82-31-787-4059

E-mail mkhan@snu.ac.kr
Dear Editor,

Immune checkpoint inhibitors have been spotlighted as a recent crucial therapy in many kinds of solid cancers. Atezolizumab is an immune checkpoint inhibitor that increases antitumor T-cell activity through binding to programmed death-ligand 1 that suppresses effector T cells. ${ }^{1}$ The potentiation of T-cell activity by atezolizumab may induce an uncontrolled immune response that can lead to a wide range of adverse events. ${ }^{2}$ One of the rare adverse reactions of atezolizumab is meningitis or encephalitis. Here we report a severe case of autoimmune encephalitis that developed after administering atezolizumab to a patient with bladder cancer.

A 49-year-old male was admitted to our hospital for status epilepticus and encephalitis of unknown origin. He was diagnosed with urothelial carcinoma of the bladder, and received the first cycle of 1,200-mg atezolizumab (Fig. 1). After administering atezolizumab he suffered general weakness and eventually showed stuporous mentality and three generalized tonic-clonic seizures upon admission. Brain MRI showed diffuse leptomeningeal enhancement. A cerebrospinal fluid (CSF) examination showed an opening pressure of $23 \mathrm{~cm} \mathrm{H}_{2} \mathrm{O}$ and that a sample of 50 white blood cells comprised 8 polymorphonuclear cells, 1 lymphocyte, and 41 cells of other types. Leptomeningeal seeding was first suspected considering his past medical history and the dominant CSF profile of the other cells. However, no malignant cells were found in CSF cytology. Antibiotics were administered since we could not fully exclude bacterial meningitis. However, the CSF profile had worsened at follow-up. We were concerned about the possibility that atezolizumab might have caused autoimmune encephalitis, and so steroid pulse therapy ( $1 \mathrm{~g}$ of methylprednisolone daily) was administered. This did not produce any improvement, and so intravenous immunoglobulin (IVIG) therapy was started. The patient initially improved clinically and follow-up MRI showed the resolution of leptomeningeal enhancement. All findings in the CSF study were negative: paraneoplastic antibodies (anti-Hu, anti-Ri, and anti-Yo antibodies), bacterial culture, fungus culture, tuberculous PCR, and viral PCR including herpes simplex virus 1 and 2, Epstein-Barr virus, varicella zoster virus, cytomegalovirus, and John Cunningham virus.

The patient experienced drowsiness about 1 month after the first IVIG therapy, so a second round of IVIG therapy was applied. However, this did not produce any further clinical improvement. There was no epileptiform discharge in electroencephalography, and the findings of a follow-up CSF study were completely normal with no malignant cells in cytology (Fig. 1). The patient had suffered persistent fever and his CRP level had seldom dropped below $20 \mathrm{mg} / \mathrm{dL}$ despite receiving antibiotics. The median onset delay for immune-related colitis in patients with urothelial carcinoma who receive atezolizumab was 1.7 months. ${ }^{3}$ The present patient was suspected as having immune-related colitis because of continuous dis-

(c) This is an Open Access article distributed under the terms of the Creative Commons Attribution Non-Commercial License (https://creativecommons.org/licenses/by-nc/4.0) which permits unrestricted non-commercial use, distribution, and reproduction in any medium, provided the original work is properly cited. 


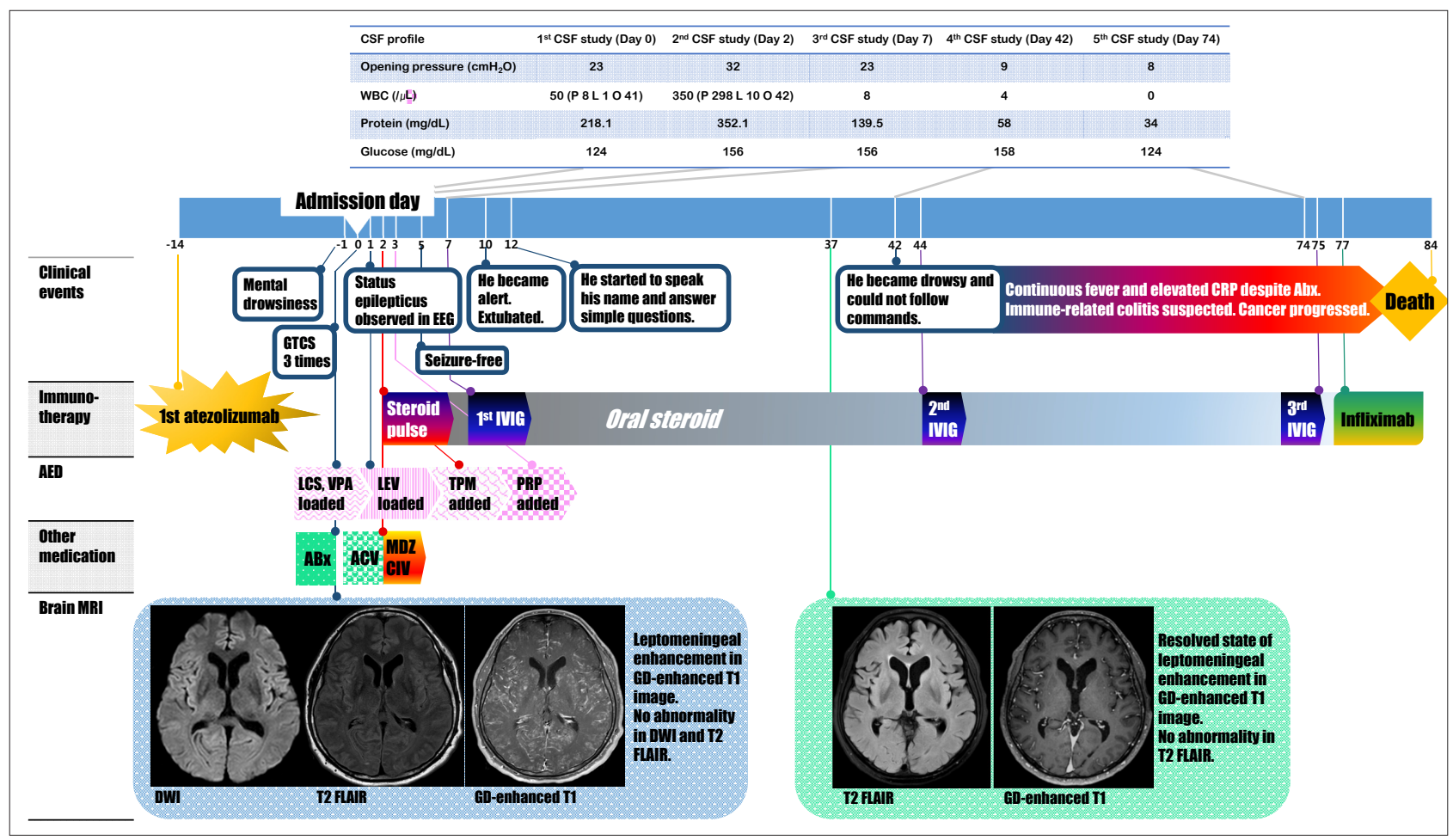

Fig. 1. Clinical course of the patient. ABx: antibiotics, ACV: acyclovir, AED: anti-epileptic drug, CRP: C-reactive protein, CSF: cerebrospinal fluid, DWI: diffusion-weighted image, EEG: electroencephalography, FLAIR: fluid-attenuated inversion recovery, GD-enhanced T1: gadolinium-enhanced T1, GTCS: generalized tonic-clonic seizure, IVIG: intravenous immunoglobulin, L: lymphocytes, LCS: lacosamide, LEV: levetiracetam, MDZ CIV: continuous intravenous infusion of midazolam, 0: other cells, P: polymorphonuclear cells, PRP: perampanel, TPM: topiramate, VPA: valproic acid, WBC: white blood cells.

tension of the colon in abdominal X-rays and Clostridiumdifficile-negative loose stools that occurred 2 months after the administration of atezolizumab. Changing from prednisolone to methylprednisolone, increasing the dose, and administering infliximab were not effective, and his cancer progressed with increased metastasis. His general condition declined and he eventually died of septic shock and multiorgan failure.

There have been several cases of autoimmune encephalopathy caused by immune checkpoint inhibitors, ${ }^{4}$ most commonly associated with ipilimumab. Three cases of autoimmune encephalopathy caused by the administration of ipilimumab and nivolumab have been reported, all of which received steroid pulse therapy and IVIG therapy. ${ }^{5-7}$ Rituximab was also administered in two of these cases. ${ }^{5,6}$ Another case of autoimmune encephalitis induced by ipilimumab and nivolumab was improved by a steroid and natalizumab. ${ }^{8}$ Two cases of atezolizumab-associated autoimmune encephalopathy improved rapidly following the administration of steroid therapy., ${ }^{9} 10$

We could not prove the direct causality of the encephalitis in the present case. Moreover, autoimmune antibodies such as anti-NMDAR, anti-AMPA, and anti-LGI1 antibodies were not checked for. However, several aspects strongly suggest that it had been triggered by atezolizumab. The patient was diagnosed with bladder cancer 1 year before administering atezolizum- ab. A paraneoplastic neurological syndrome is rare in bladder cancer ${ }^{11}$ and usually precedes its diagnosis. ${ }^{12}$ The rapid progression of neurological symptoms after only a single dose of atezolizumab and the neurological improvement after administering immunosuppressive therapy suggests that the disease course was far from a paraneoplastic syndrome. In addition, the patient was suspected as having immune-related colitis, which is not expected in other types of autoimmune encephalitis.

It would be reasonable to attempt immunosuppressive therapy such as steroid pulse therapy and IVIG therapy: the former might suppress autoimmune T-cell activity and the latter might help neutralizing the immune checkpoint inhibitor. Our case suggests that IVIG should be considered in atezolizumab-associated encephalopathy that does not respond significantly to steroid therapy. Besides, the half-life of atezolizumab is 27 days, ${ }^{13}$ and it was on the 27 th day after the administration of atezolizumab when the patient started to show clinical improvement. We therefore suggest that immunosuppressive therapy was effective not only against the condition itself, but also helped with the natural decrement of atezolizumab. Additional IVIG therapy could be considered in cases of aggravation of the neurological status, but this did not bring any improvement in our case. Perhaps natalizumab 
ties of anti-cancer immune checkpoint blockade. J Neurol Neuromed 2016;1:39-45

5. Ito M, Fujiwara S, Fujimoto D, Mori R, Yoshimura H, Hata A, et al. Rituximab for nivolumab plus ipilimumab-induced encephalitis in a small-cell lung cancer patient. Ann Oncol 2017;28:2318-2319.

6. Williams TJ, Benavides DR, Patrice KA, Dalmau JO, De Ávila AL, Le DT, et al. Association of autoimmune encephalitis with combined immune checkpoint inhibitor treatment for metastatic cancer. JAMA Neurol 2016;73:928-933.

7. Maurice C, Schneider R, Kiehl TR, Bavi P, Roehrl MH, Mason WP, et al. Subacute CNS demyelination after treatment with nivolumab for melanoma. Cancer Immunol Res 2015;3:1299-1302.

8. Hottinger AF, De Micheli R, Guido V, Karampera A, Hagmann P, Du Pasquier R. Natalizumab may control immune checkpoint inhibitorinduced limbic encephalitis. Neurol Neuroimmunol Neuroinflamm 2018;5:e439.

9. Levine JJ, Somer RA, Hosoya H, Squillante C. Atezolizumab-induced encephalitis in metastatic bladder cancer: a case report and review of the literature. Clin Genitourin Cancer 2017;15:e847-e849.

10. Laserna A, Tummala S, Patel N, El Hamouda DEM, Gutiérrez C. Atezolizumab-related encephalitis in the intensive care unit: case report and review of the literature. SAGE Open Med Case Rep 2018;6: $2050313 X 18792422$.

11. Sacco E, Pinto F, Sasso F, Racioppi M, Gulino G, Volpe A, et al. Paraneoplastic syndromes in patients with urological malignancies. Urol Int 2009;83:1-11.

12. Darnell RB, Posner JB. Paraneoplastic syndromes involving the nervous system. N Engl J Med 2003;349:1543-1554.

13. Stroh M, Winter H, Marchand M, Claret L, Eppler S, Ruppel J, et al. Clinical pharmacokinetics and pharmacodynamics of atezolizumab in metastatic urothelial carcinoma. Clin Pharmacol Ther 2017;102:305312.

4. Blackmon JT, Viator TM, Conry RM. Central nervous system toxici- 